\title{
Optimal adaptive control for a class of stochastic systems
}

\author{
ARUNABHA BAGCHI ${ }^{1}$ and HAN-FU CHEN $^{2}$ \\ 'Department of Applied Mathematics, University of Twente, PO Box 217,7500 \\ AE Enschede, The Netherlands \\ ${ }^{2}$ Institute of Systems Science, Chinese Academy of Sciences, Beijing 100080 , \\ People's Republic of China \\ e-mail: a.bagchi@math.utwente.nl
}

\begin{abstract}
We study linear-quadratic adaptive tracking problems for a special class of stochastic systems expressed in the state-space form. This is a long-standing problem in the control of aircraft flying through atmospheric turbulence. Using an ELS-based algorithm and introducing dither in the control law we show that the resulting control achieves optimal cost in the limit, while simultaneously the unknown parameters converge to their true values.
\end{abstract}

Keywords. Stochastic adaptive control; tracking problem; ELS-based estimator.

\section{Introduction}

There is an enormous amount of literature on stochastic adaptive control starting with the pioneering work of Åström \& Wittenmark (1973) on the self-tuning regulator. Most of the research in this area, however, concentrated on ARMAX models (Kumar 1985). Parallel to this was the somewhat unrelated development of the design of adaptive flight control systems, starting with the thesis of Illiff (1973). Most researchers in this area start with the dynamical model of aircraft in flight and, consequently, formulate their problem in the state-space form. Unfortunately, the literature on stochastic adaptive control for systems in state-space form is rather limited. Kumar (1983) made a thorough analysis on the problem of controlling an unknown linear-Gaussian system with quadratic criterion but had to restrict himself to the case of complete observation of the system states. We study here the problem of controlling a linear system with incomplete and inaccurate observation of the system states so that a quadratic tracking criterion is minimized in the situation when the matrix multiplying the control term in the state equation is unknown. We do not assume that the observation noise is Gaussian, but do restrict ourselves to the situation with no state noise. This problem arises naturally in controlling the flight of an aircraft in atmospheric turbulence where the objective is to minimize the normal acceleration or gust response in the angle of attack (Wang 1993). This is done in order to improve passenger 
and pilot comfort. It corresponds to our problem when the so-called control derivatives of the aircraft are unknown.

\section{Problem formulation}

Consider the following discrete-time dynamical system

$$
\begin{aligned}
x_{k+1} & =A x_{k}+B u_{k}, \\
y_{k} & =C x_{k}+w_{k},
\end{aligned}
$$

where $x_{k}$ and $y_{k}$, for fixed $k$, are $\mathbb{R}^{n}$ - and $\mathbb{R}^{m}$-valued state and observation vectors respectively; $u_{k}$ is an $\mathbb{R}^{p}$-valued control vector, $\left\{w_{k}\right\}$ is a noise sequence to be specified below, the matrices $A$ and $C$ are known, while the matrix $B$ is unknown. Our objective is to minimize the tracking criterion

$$
J=\lim _{N \rightarrow \infty} \sup \frac{1}{N} J_{N}(u),
$$

where

$$
J_{N}(u) \triangleq \sum_{k=0}^{N-1}\left[\left(x_{k}-x_{k}^{*}\right)^{\prime} Q_{1}\left(x_{k}-x_{k}^{*}\right)+u_{k}^{\prime} Q_{2} u_{k}\right],
$$

with $Q_{1} \geq 0, Q_{2}>0$ and $\left\{x_{k}^{*}\right\}$ a prescribed sequence of desired path.

We follow the self-tuning approach which is based on the certainty equivalence principle. Thus we first determine the optimal control law assuming that $B$ is known, and then replace $B$ by an appropriate on-line estimator. Recursive estimator for $B$ may be obtained by using extended least squares (ELS), maximum likelihood (ML), stochastic approximation (SA) or Kalman filter methods. In the specific problem considered here, the Kalman filter method yields readily a recursive estimator for $B$ (Balakrishnan 1987). The estimator also has been proved (Balakrishnan 1987) to converge to the true value in the mean-square sense. When we close the control loop the analysis becomes more complicated. Asymptotic optimality of the resulting control law has not been established yet. One difficulty is that the estimator loses the interpretation of being the conditional espectation when the system operates in closed loop. We propose in the next section an ELS-based method to estimate $B$.

The rest of this section is devoted to determining the optimal control law when $B$ is known. For this, we make the following assumptions:

A1. $\alpha^{-1}\left(e^{i \lambda}\right)+\alpha^{-1}\left(e^{-i \lambda}\right)-1>0 \forall \lambda \in[0,2 \pi)$, where $\alpha(z) \triangleq \operatorname{det}(I-z A) \equiv 1+$ $a_{1} z+\cdots+a_{n} z^{n}, z \in \mathbb{C}$. This is the strict positive real (SPR) condition. Note that this condition implies $\alpha(z) \neq 0 \forall|z| \leq 1$ (see Chen \& Guo 1991, corollary 4.1).

A2. $(A, C)$ is observable.

A3. $(A, B)$ is controllable and $(A, D)$ is observable, where $D$ is any matrix satisfying $D^{\prime} D=Q_{1}$.

A4. $\left\{w_{k}, \mathcal{F}_{k}\right\}$ is a martingale difference sequence (mds) with 


$$
\lim _{k \rightarrow \infty} \sup E\left[\left\|w_{k}\right\|^{\beta} \mid J_{k-1}\right]<\infty \quad \text { a.s. for some } \beta \geq 2 \text {. }
$$

The calculations that follow are well-known (see Bagchi 1993, for example). They are included only for completeness. By A3, there is a unique solution to the following algebraic Riccati equation in the class of positive definite matrices.

$$
S=A^{\prime} S A-A^{\prime} S B\left(Q_{2}+B^{\prime} S B\right)^{-1} B^{\prime} S A+Q_{1}
$$

and the matrix,

$$
F \triangleq A-B\left(Q_{2}+B^{\prime} S B\right)^{-1} B^{\prime} S A,
$$

is asymptotically stable. Define

$$
\begin{aligned}
& L \triangleq-\left(Q_{2}+B^{\prime} S B\right)^{-1} B^{\prime} S A \\
& b_{k} \triangleq-\sum_{j=0}^{\infty}\left(F^{j}\right)^{\prime} Q_{1} x_{k+j}^{*}=F^{\prime} b_{k+1}-Q_{1} x_{k}^{*} \\
& d_{k} \triangleq-\left(Q_{2}+B^{\prime} S B\right)^{-1} B^{\prime} b_{k+1} .
\end{aligned}
$$

Using (5), we have,

$$
\begin{aligned}
x_{N}^{\prime} S x_{N}-x_{0}^{\prime} S x_{0}= & \sum_{k=0}^{N-1}\left(x_{k+1}^{\prime} S x_{k+1}-x_{k}^{\prime} S x_{k}\right) \\
= & \sum_{k=0}^{N-1}\left(A x_{k}+B u_{k}\right)^{\prime} S\left(A x_{k}+B u_{k}\right) \\
& -\sum_{k=0}^{N-1} x_{k}^{\prime}\left[A^{\prime} S A-A^{\prime} S B\left(Q_{2}+B^{\prime} S B\right)^{-1} B^{\prime} S A+Q_{1}\right] x_{k},
\end{aligned}
$$

so that

$$
\begin{aligned}
& \sum_{k=0}^{N-1}\left(x_{k}^{\prime} Q_{1} x_{k}+u_{k}^{\prime} Q_{2} u_{k}\right) \\
& =x_{0}^{\prime} S x_{0}-x_{N}^{\prime} S x_{N}+\sum_{k=0}^{N-1}\left(u_{k}^{\prime} B^{\prime} S B u_{k}+2 x_{k}^{\prime} A^{\prime} S B u_{k}\right. \\
& \left.\quad+x_{k}^{\prime} L^{\prime}\left(Q_{2}+B^{\prime} S B\right) L x_{k}+u_{k}^{\prime} Q_{2} u_{k}\right)
\end{aligned}
$$

Similarly, we obtain

$$
\begin{aligned}
b_{N}^{\prime} x_{N}-b_{0}^{\prime} x_{0} & =\sum_{k=0}^{N-1}\left(b_{k+1}^{\prime} x_{k+1}-b_{k}^{\prime} x_{k}\right) \\
& =\sum_{k=0}^{N-1}\left[b_{k+1}^{\prime}\left(A x_{k}+B u_{k}\right)-b_{k}^{\prime} x_{k}\right] .
\end{aligned}
$$


Using (10) and (11), we get

$$
\begin{aligned}
J_{N}(u)= & \sum_{k=0}^{N-1}\left(x_{k}^{\prime} Q_{1} x_{k}-2 x_{k}^{*^{\prime}} Q_{1} x_{k}+x_{k}^{*^{\prime}} Q_{1} x_{k}^{*}+u_{k}^{\prime} Q_{2} u_{k}\right) \\
= & x_{0}^{\prime} S x_{0}-x_{N}^{\prime} S x_{N}+\sum_{k=0}^{N-1}\left(u_{k}^{\prime} B^{\prime} S B u_{k}+2 x_{k}^{\prime} A^{\prime} S B u_{k}\right. \\
& \left.+x_{k}^{\prime} L^{\prime}\left(Q_{2}+B^{\prime} S B\right) L x_{k}+u_{k}^{\prime} Q_{2} u_{k}\right)+2 b_{0}^{\prime} x_{0}-2 b_{N}^{\prime} x_{N} \\
& +2 \sum_{k=0}^{N-1}\left[b_{k+1}^{\prime}\left(A x_{k}+B u_{k}\right)-b_{k}^{\prime} x_{k}\right]+\sum_{k=0}^{N-1}\left(x_{k}^{*^{\prime}} Q_{1} x_{k}^{*}-2 x_{k}^{*^{\prime}} Q_{1} x_{k}\right)
\end{aligned}
$$

From (8) we get

$$
b_{k}^{\prime} x_{k}+x_{k}^{*^{\prime}} Q_{1} x_{k}=b_{k+1}^{\prime} F x_{k}=b_{k+1}^{\prime} A x_{k}+b_{k+1}^{\prime} B L x_{k} .
$$

Putting this in (12) yields

$$
\begin{aligned}
J_{N}(u)= & x_{0}^{\prime} S x_{0}-x_{N}^{\prime} S x_{N}+2_{0}^{\prime} x_{0}-2 b_{N}^{\prime} x_{N} \\
& +\sum_{k=0}^{N-1}\left(u_{k}^{\prime} B^{\prime} S B u_{k}+2 x_{k}^{\prime} A^{\prime} S B u_{k}+u_{k}^{\prime} Q_{2} u_{k}\right. \\
& \left.+x_{k}^{\prime} L^{\prime}\left(Q_{2}+B^{\prime} S B\right) L x_{k}+2 b_{k+1}^{\prime} B u_{k}-2 b_{k+1}^{\prime} B L x_{k}+x_{k}^{*^{\prime}} Q_{1} x_{k}^{*}\right) \\
= & x_{0}^{\prime} S x_{0}-x_{N}^{\prime} S x_{N}+2 b_{0}^{\prime} x_{0}-2 b_{N}^{\prime} x_{N}+\sum_{k=0}^{N-1}\left(x_{k}^{*^{\prime}} Q_{1} x_{k}^{*}-d_{k}^{\prime} B^{\prime} b_{k+1}\right) \\
& +\sum_{k=0}^{N-1}\left(u_{k}-L x_{k}-d_{k}\right)^{\prime}\left(Q_{2}+B^{\prime} S B\right)\left(u_{k}-L x_{k}-d_{k}\right)
\end{aligned}
$$

Let $\mathcal{U}$ be the class of admissible controls which will be specified below. The point to note at this moment is that, whatever class $\mathcal{U}$ we choose for admissible controls,

$$
\inf _{u \in \mathcal{U}} \cdot \lim _{N \rightarrow \infty} \sup \frac{1}{N} J_{N}(u) \geq \lim _{N \rightarrow \infty} \sup \frac{1}{N} \sum_{k=0}^{N-1}\left[x_{k}^{*^{\prime}} Q_{1} x_{k}^{*}-d_{k}^{\prime} B^{\prime} b_{k+1}\right]
$$

Thus, if in the class of desired control laws we are able to choose a control for which $\lim _{N \rightarrow \infty} \sup (1 / N) J_{N}(u)$ equals the right hand side of (15), then this will automatically yield the optimal control desired.

\section{Recursive estimator for $B$}

Let

$$
C \operatorname{adj}(I-z A) \equiv C+C_{1} z+\cdots+C_{n-1} z^{n-1}
$$


where "adj" stands for the adjoint of a matrix and $C_{i}$ are $m \times n$ matrices, $i=1, \ldots, n-1$. Set

$$
\theta^{\prime}=\left[C B \vdots C_{1} B \vdots \ldots \vdots C_{n-1} B\right]
$$

and

$$
\phi_{k}^{\prime}=\left[u_{k}^{\prime}: u_{k-1}^{\prime} \vdots \ldots: u_{k-n+1}^{\prime}\right]
$$

where $C B$ and $C_{i} B$ are $m \times p$ matrices.

Let us define

$$
\Sigma \triangleq\left[\begin{array}{c}
C \\
C_{1} \\
\vdots \\
C_{n-1}
\end{array}\right] \text { and } \Pi=\left[\begin{array}{c}
C B \\
C_{1} B \\
\vdots \\
C_{n-1} B
\end{array}\right]
$$

Lemma 1. With the notations above,

$$
B=\left(\Sigma^{\prime} \Sigma\right)^{-1} \Sigma^{\prime} \Pi \text {. }
$$

Proof. All we have to show is that $\Sigma$ is of full row-rank so that $\Sigma^{\prime} \Sigma$ is invertible. Suppose that

$$
\Sigma x=0, \quad x \in \mathbb{R}^{n}
$$

Then

$$
C \operatorname{adj}(I-z A) x=0
$$

which implies that

$$
C(I-z A)^{-1} x=0 .
$$

Then for $|z|$ sufficiently small,

$$
C\left(1+z A+z^{2} A^{2}+\cdots\right) x \equiv 0 .
$$

From this we conclude that

$$
C x=0, \quad C A x=0, \ldots, C A^{n} x=0 .
$$

Since $(A, C)$ is observable, we must have $x=0$, establishing that $\Sigma$ is of full row rank.

We are now in a position to propose a recursive algorithm for estimating $B$. We first propose the following scheme to estimate $\theta$ recursively:

$$
\begin{aligned}
\theta_{k+1} & =\theta_{k}+\gamma_{k} P_{k} \phi_{k}\left[\alpha(z) y_{k+1}-\theta_{k}^{\prime} \phi_{k}-(\alpha(z)-1) \hat{w}_{k+1}\right], \\
P_{k+1} & =P_{k}-\gamma_{k} P_{k} \phi_{k} \phi_{k}^{\prime} P_{k} \\
\gamma_{k} & =\left(1+\phi_{k}^{\prime} P_{k} \phi_{k}\right)^{-1} \\
\hat{w}_{k+1} & =\alpha(z) y_{k+1}-\theta_{k+1}^{\prime} \phi_{k}-(\alpha(z)-1) \hat{w}_{k+1},
\end{aligned}
$$

where $z$ now stands for the unit delay operator and $\theta_{0}, P_{0}, \hat{w}_{0}$ are chosen arbitrarily. 
Let us write $\hat{\theta}_{k}$ in the block matrix form

$$
\theta_{k}^{\prime}=\left[\theta_{k 1} \vdots \ldots \vdots \theta_{k n}\right]
$$

where $\theta_{k i}$ are $m \times p$ matrices, $i=1, \ldots, n$, and set

$$
\Pi_{k}=\left[\begin{array}{c}
\theta_{k 1} \\
\vdots \\
\theta_{k n}
\end{array}\right]
$$

We propose the following recursive estimator for $B$ :

$$
B_{k}=\left(\Sigma^{\prime} \Sigma\right)^{-1} \Sigma^{\prime} \Pi_{k}
$$

Theorem 1. Assume that conditions A1, A2 and A4 hold. Then for any $\mathcal{F}_{k}$-measurable control $u_{k}$,

$$
\left\|B-B_{k+1}\right\|^{2}=O\left(\frac{\log \lambda_{\max }(k)\left(\log \log \lambda_{\max }(k)\right)^{\Delta(\beta-2)}}{\lambda_{\min }(k)}\right)
$$

where $\lambda_{\max }(k)$ and $\lambda_{\min }(k)$ denote the maximum and minimum eigenvalues of $P_{k+1}^{-1}$, respectively, $\beta$ is as defined in $\mathrm{A} 4$ and

$$
\Delta(\beta-2)=\left\{\begin{array}{l}
0 \text { if } \beta>2 \\
c>1, \text { but otherwise arbitrary, if } \beta=2 .
\end{array}\right.
$$

Proof. Using lemma 1, we have

$$
B-B_{k}=\left(\Sigma^{\prime} \Sigma\right)^{-1} \Sigma^{\prime}\left(\Pi-\Pi_{k}\right),
$$

so that it is sufficient for us to show that

$$
\left\|\theta-\theta_{k+1}\right\|^{2}=O\left(\frac{\log \lambda_{\max }(k)\left(\log \log \lambda_{\max }(k)\right)^{\Delta(\beta-2)}}{\lambda_{\min }(k)}\right) .
$$

Note that, with $z$ denoting the unit delay operator, we may rewrite (1) and (2) symbolically as follows:

$$
\begin{aligned}
& x_{k}=(I-z A)^{-1} B u_{k-1}, \\
& y_{k}=C(I-z A)^{-1} B u_{k-1}+w_{k} .
\end{aligned}
$$

Therefore, we get

$$
\alpha(z) y_{k}=C \operatorname{adj}(I-z A) B u_{k-1}+\alpha(z) w_{k} .
$$

Using (17) and (18), we may express (28) as

$$
\alpha(z) y_{k+1}=\theta^{\prime} \phi_{k}+\alpha(z) w_{k+1} .
$$

Let us denote

$$
\xi_{k+1} \triangleq \hat{w}_{k+1}-w_{k+1} .
$$


Using (23) and (29), we have

$$
\begin{aligned}
\xi_{k+1} & =\theta^{\prime} \phi_{k}+\alpha(z) w_{k+1}-\theta_{k+1}^{\prime} \phi_{k}-(\alpha(z)-1) \hat{w}_{k+1}-w_{k+1} \\
& =\tilde{\theta}_{k+1}^{\prime} \phi_{k}-(\alpha(z)-1) \xi_{k+1}
\end{aligned}
$$

or, equivalently,

$$
\alpha(z) \xi_{k+1}=\tilde{\theta}_{k+1}^{\prime} \phi_{k}
$$

where $\tilde{\theta}_{k+1} \triangleq \theta-\theta_{k+1}$. Using (20) and (23) we have

$$
\begin{aligned}
\hat{w}_{k+1}= & \xi_{k+1}+w_{k+1}=\alpha(z) y_{k+1}-(\alpha(z)-1) \hat{w}_{k+1} \\
& -\left(\theta_{k}+\gamma_{k} P_{k} \phi_{k}\left(\alpha(z) y_{k+1}-\theta_{k}^{\prime} \phi_{k}-(\alpha(z)-1) \hat{w}_{k+1}\right)^{\prime}\right)^{\prime} \phi_{k} \\
= & \left(1-\gamma_{k} \phi_{k}^{\prime} P_{k} \phi_{k}\right)\left(\alpha(z) y_{k+1}-(\alpha(z)-1) \hat{w}_{k+1}-\theta_{k}^{\prime} \phi_{k}\right) \\
= & \gamma_{k}\left(\alpha(z) y_{k+1}-(\alpha(z)-1) \hat{w}_{k+1}-\theta_{k}^{\prime} \phi_{k}\right) .
\end{aligned}
$$

Therefore, (20) may be rewritten as

$$
\theta_{k+1}=\theta_{k}+P_{k} \phi_{k}\left(\xi_{k+1}+w_{k+1}\right)^{\prime}
$$

or, equivalently,

$$
\tilde{\theta}_{k+1}=\tilde{\theta}_{k}-P_{k} \phi_{k}\left(\xi_{k+1}+w_{k+1}\right)^{\prime} .
$$

The result now follows from theorem 2 of Chen \& Guo (1991) by identifying $\eta_{k}$ in that theorem with,

$$
\alpha(z) y_{k+1}-(\alpha(z)-1) \hat{w}_{k+1}-\theta_{k}^{\prime} \phi_{k}-w_{k+1},
$$

which is easily seen to be $\mathcal{F}_{k}$-measurable.

\section{Consistent estimator for $B$}

The previous theorem shows that the estimation error of $B_{k}$ depends upon the behavior of $P_{k}^{-1}$. In general, we do not know whether $B_{k}$ converges to the true $B$ or not. To ensure strong consistency of the estimator of unknown parameters and achieve optimality of the control law at the same time is a very difficult problem. Direct certainty-equivalence based adaptive contol law cannot achieve this goal in the linear-quadratic problem (Polderman 1989). In stochastic adaptive control literature the idea of diminishing dither to the control law has been introduced for this purpose (Caines \& Lafortune 1984; Chen 1984), which will be used here.

Let $\left\{v_{k}\right\}$ be a sequence of $\mathbb{R}^{p}$-valued random vectors which is independent of $\left\{w_{k}\right\}$, with $E v_{k}=0, E v_{k} v_{k}^{\prime}=I$ and $\left\|v_{k}\right\| \leq$ constant a.s. (almost surely). Define

$$
u_{k}^{d} \triangleq \frac{v_{k}}{k^{\epsilon / 2}}, \quad \epsilon \in\left[0, \frac{1}{2 n}\right) .
$$


Without loss of generality, we may assume that $\mathcal{F}_{k} \equiv \sigma\left\{w_{i}, v_{i}, 0 \leq i \leq k\right\}$. Set

$$
\mathcal{F}_{k}^{\prime} \equiv \sigma\left\{w_{i}, 0 \leq i \leq k, \quad v_{j}, \quad 0 \leq j \leq k-1\right\} .
$$

Let $u_{k}^{s}$ be any $\mathcal{F}_{k}^{\prime}$-measurable control law at time $k$, obtained possibly by some certainty equivalence principle. We apply the diminishingly excited version $u_{k}$ of $u_{k}^{s}$ to the system,

$$
u_{k}=u_{k}^{s}+u_{k}^{d}
$$

Theorem 2. If $\mathrm{A} 1, \mathrm{~A} 2, \mathrm{~A} 4$ hold, and if

$$
\sum_{i=1}^{k}\left\|u_{i}^{s}\right\|^{2}=O\left(k^{1+\delta}\right), \quad \delta \in\left[0, \frac{1-2 \epsilon n}{1+2 n}\right), \quad \text { a.s. }
$$

Then

$$
\begin{gathered}
\left\|B-B_{k}\right\|^{2}=O\left(\frac{\log k(\log \log k)^{\Delta(\beta-2)}}{k^{\alpha}}\right), \\
\alpha \in\left(\frac{1}{2}(1+\delta), 1-n(\epsilon+\delta)\right] .
\end{gathered}
$$

Proof. We first note that the interval for $\alpha$ is not empty, because

$$
n(\epsilon+\delta)+\frac{\delta}{2}<n\left(\epsilon+\frac{1-2 \epsilon n}{1+2 n}\right)+\frac{1-2 \epsilon n}{2(1+2 n)}=\frac{1}{2} .
$$

It is easy to establish (see equation (6.72) in Chen \& Guo 1991) that

$$
\frac{1-\epsilon}{k^{1-\epsilon}} \sum_{i=1}^{k} u_{k}^{d} u_{k}^{d^{\prime}} \rightarrow I \text { as } n \rightarrow \infty
$$

Then

$$
\begin{aligned}
\lambda_{\max }(k) & =O\left(\sum_{i=1}^{k}\left\|u_{i}\right\|^{2}\right) \\
& =O\left(\sum_{i=1}^{k}\left(\left\|u_{i}^{s}\right\|^{2}+\left\|u_{i}^{d}\right\|^{2}\right)\right)=O\left(k^{1+\delta}\right) .
\end{aligned}
$$

Using (26) and (38), we can establish (36) provided that

$$
\lim _{k \rightarrow \infty} \inf k^{-\alpha} \lambda_{\min }\left(\sum_{i=0}^{k} \phi_{i} \phi_{i}^{\prime}\right) \neq 0
$$

where $\lambda_{\min }(X)$ denotes the minimum eigenvalue of a matrix $X$. Suppose that (39) does not hold. Then there exists a sequence $\left\{\zeta_{k_{\ell}}\right\}$ with

$$
\zeta_{k_{\ell}}=\left[\rho_{k_{\ell}}^{(1)^{\prime}} \vdots \ldots: \rho_{k_{\ell}}^{(n)^{\prime}}\right]^{\prime}, \quad\left\|\zeta_{k_{\ell}}\right\|=1
$$


$\rho_{k_{\ell}}^{(i)}$ being $p$-dimensional vectors, such that

$$
\lim _{\ell \rightarrow \infty} k_{\ell}^{-\alpha} \sum_{i=1}^{k_{\ell}}\left(\zeta_{k_{\ell}}^{\prime} \phi_{i}\right)^{2}=0
$$

or, equivalently,

$$
\lim _{\ell \rightarrow \infty} k_{\ell}^{-\alpha} \sum_{i=1}^{k_{\ell}}\left(\rho_{k_{\ell}}^{(1)^{\prime}} u_{i}+\cdots+\rho_{k_{\ell}}^{(n)^{\prime}} u_{i-n+1}\right)^{2}=0
$$

By theorem 2.8 of Chen \& Guo (1991),

$$
\begin{aligned}
& \left\|\sum_{i=1}^{k} u_{i-j}^{s} u_{i}^{d^{\prime}}\right\|=O\left(k^{(1+\delta / 2)}(\log k)^{(1 / 2)+\eta}\right), \quad \forall j \geq 0, \quad \forall \eta>0, \\
& \left\|\sum_{i=1}^{k} u_{i-j} u_{i}^{d^{\prime}}\right\|=O\left(k^{(1+\delta / 2)}(\log k)^{(1 / 2)+\eta}\right), \quad \forall j>0 .
\end{aligned}
$$

Since $\alpha>(1+\delta) / 2$, we then have,

$$
k_{\ell}^{-\alpha}\left\{\rho_{k_{\ell}}^{(1)^{\prime}} \sum_{i=1}^{k_{\ell}} u_{i}^{s} u_{i}^{d} \rho_{k_{\ell}}^{(1)}+\sum_{j=2}^{n} \rho_{k_{\ell}}^{(j)^{\prime}} \sum_{i=1}^{k_{\ell}} u_{i-j+1} u_{i}^{d^{\prime}} \rho_{k_{\ell}}^{(1)}\right\} \rightarrow 0, \quad k \rightarrow \infty,
$$

which, using (40), leads to

$$
k_{\ell}^{-\alpha} \sum_{i=1}^{k_{\ell}}\left(\rho_{k_{\ell}}^{(1)^{\prime}} u_{i}^{d}\right)^{2} \rightarrow 0, k \rightarrow \infty
$$

and

$$
k_{\ell}^{-\alpha} \sum_{i=1}^{k_{\ell}}\left(\rho_{k_{\ell}}^{(1)^{\prime}} u_{i}^{s}+\rho_{k_{\ell}}^{(2)^{\prime}} u_{i-1}+\cdots+\rho_{k_{\ell}}^{(n)^{\prime}} u_{i-n+1}\right)^{2} \rightarrow 0, \quad k \rightarrow \infty .
$$

From (37) and (41) it follows that

$$
\left\|\rho_{k_{\ell}}^{(1)}\right\|^{2}=o\left(k_{\ell}^{-(1-\epsilon-\alpha)}\right) \text {, }
$$

which, together with (35), implies that

$$
k_{\ell}^{-(I+\delta)+1-\epsilon-\alpha} \sum_{i=1}^{k_{\ell}}\left(\rho_{k_{\ell}}^{(1)^{\prime}} u_{i}^{s}\right)^{2} \rightarrow 0, \quad k \rightarrow \infty .
$$

Equations (44) and (42) imply that

$$
k_{\ell}^{-\alpha-(\epsilon+\delta)} \sum_{i=1}^{k_{\ell}}\left(\rho_{k_{\ell}}^{(2)^{\prime}} u_{i-1}+\cdots+\rho_{k_{\ell}}^{(n)^{\prime}} u_{i-n+1}\right)^{2} \rightarrow 0, \quad k \rightarrow \infty .
$$

Comparing (45) with (40) yields

$$
\left\|\rho_{k_{\imath}}^{(i)}\right\|^{2}=o\left(k_{\ell}^{-(1-\epsilon-\alpha-(i-1)(\epsilon+\delta))}\right), \quad i=1, \ldots, n .
$$


Noticing that $\alpha \leq 1-n(\epsilon+\delta)$, we find that for every $i, 1 \leq i \leq n$,

$$
\alpha \leq 1-i(\epsilon+\delta)=1-\epsilon-\delta-(i-1)(\epsilon+\delta)<1-\epsilon-(i-1)(\epsilon+\delta) .
$$

Therefore, (46) implies that

$$
\rho_{k_{\ell}}^{(i)} \rightarrow 0, \quad \ell \rightarrow \infty, \quad i=1, \ldots, n .
$$

This contradicts our assumption that $\left\|\rho_{k_{\ell}}\right\|=1$ for all $\ell$. This, in turn, contradicts (40) establishing our result.

\section{Optimal adaptive control}

Let us now go back to the adaptive control problem posed in $\$ 2$. It is clear from (14) that, if $\left\{x_{k}\right\}$ was completely observed and $\left\{B_{k}\right\}$ was known, the optimal control would be given by

$$
u_{k}=L x_{k}+d_{k} \text {. }
$$

We use the ELS-based estimator $B_{k}$ for $B$ and define the certainty-equivalence control $u^{0}$ by

$$
u_{k}^{0}=L_{k} \hat{x}_{k}+\hat{d}_{k},
$$

where

$$
\hat{x}_{k+1}=A \hat{x}_{k}+B_{k} u_{k}^{0}
$$

$\hat{x}_{0}$ arbitrary,

$$
\begin{aligned}
& L_{k}=-\left(Q_{2}+B_{k}^{\prime} S_{k} B_{k}\right)^{-1} B_{k}^{\prime} S_{k} A, \\
& S_{k}=A^{\prime} S_{k-1} A-A^{\prime} S_{k-1} B_{k}\left(Q_{2}+B_{k}^{\prime} S_{k-1} B_{k}\right)^{-1} B_{k}^{\prime} S_{k-1} A+Q_{1},
\end{aligned}
$$

$S_{0} \geq 0$, otherwise arbitrary,

$$
\begin{aligned}
& \hat{d}_{k}=-\left(Q_{2}+B_{k}^{\prime} S_{k} B_{k}\right)^{-1} B_{k} \hat{b}_{k+1}, \\
& \hat{b}_{k}=-\sum_{j=0}^{k} F_{k-1}^{j} Q_{1} x_{k+j}^{*}, \\
& F_{k}=A-B_{k} L_{k} .
\end{aligned}
$$

We now define stopping times $\left\{\sigma_{k}\right\}$ and $\left\{\tau_{k}\right\}$ as follows: Set $\tau_{1}=1$. Let

$$
\begin{aligned}
\sigma_{k} & =\sup \left\{t>\tau_{k} \mid \sum_{i=\tau_{k}}^{j-1}\left\|u_{i}^{0}\right\|^{2} \leq(j-1)^{1+\delta}+\left\|u_{\tau_{k}}^{0}\right\|^{2}, \forall j \epsilon\left(\tau_{k}, t\right)\right\}, \\
\tau_{k+1} & =\inf \left\{t>\sigma_{k} \mid \sum_{i=\tau_{k}}^{\sigma_{k}-1}\left\|u_{i}^{0}\right\|^{2} \leq \frac{t^{1+\delta}}{2^{k}} \bigwedge \sum_{i=\sigma_{k}}^{t}\left\|u_{i}^{0}\right\|^{2} \leq \frac{t^{1+\delta}}{2^{k}}\right\} .
\end{aligned}
$$


The desired control law $u_{k}^{s}$ is defined by

$$
u_{k}^{s}= \begin{cases}u_{k}^{0}, & \text { if } k \epsilon\left(\tau_{\ell}, \sigma_{\ell}\right) \text { for some } \ell \\ 0, & \text { otherwise }\end{cases}
$$

and finally, the adaptive control law we are after is given by

$$
u_{k}^{*}=u_{k}^{s}+u_{k}^{d} \text {. }
$$

Let $\mathcal{U}$ denote the class of admissible controls defined by

$$
\begin{aligned}
& \mathcal{U}=\left\{u \mid u_{k} \text { is } \mathcal{F}_{k}-\right.\text { measurable such that the } \\
& \text { resulting state satisfies } \left.\left\|x_{k}\right\|^{2}=o(k) a . s .\right\}
\end{aligned}
$$

Theorem 3. Assume that $\mathrm{A} 1-\mathrm{A} 4$ hold. Then $\left\{u_{k}^{*}\right\} \in \mathcal{U}$,

$$
\lim _{N \rightarrow \infty} \sup \frac{1}{N} J_{N}\left(u^{*}\right)=\lim _{N \rightarrow \infty} \sup \frac{1}{N} \sum_{k=0}^{N-1}\left[x_{k}^{*^{\prime}} Q x_{k}^{*}-d_{k}^{\prime} B^{\prime} b_{k+1}\right]
$$

and

$$
\left\|B-B_{k}\right\|^{2}=O\left(\frac{\log k(\log \log k)^{\Delta(\beta-2)}}{k^{\alpha}}\right), \quad \alpha \in\left(\frac{1}{2}, 1-n \epsilon\right]
$$

Proof. We first show consistency of $B_{k}$. If $\tau_{\ell}<\infty$ and $\sigma_{\ell}=\infty$ for some $\ell$, then $\sum_{i=1}^{k}\left\|u_{i}^{s}\right\|^{2}=O\left(k^{1+\delta}\right)$ and the strong consistency of $B_{k}$ follows from theorem 2. If $\sigma_{\ell}<\infty$ and $\tau_{\ell+1}=\infty$ for some $\ell$, then $u_{i}^{s}=0 \forall i \geq \ell$ and, again by theorem $2, B_{k}$ is strongly consistent.

Consider now that $\tau_{\ell}<\infty, \sigma_{\ell}<\infty$ for all $\ell$. Then

$$
\begin{aligned}
\sup _{\tau_{\ell \leq k<\tau_{\ell+1}}} \frac{1}{k^{1+\delta}} \sum_{i=1}^{k}\left\|u_{i}^{s}\right\|^{2} & =\sup _{\tau_{\ell} \leq k<\sigma_{\ell}} \frac{1}{k^{1+\delta}} \sum_{i=1}^{k}\left\|u_{i}^{0}\right\|^{2} \\
& =\sup _{\tau_{\ell} \leq k<\sigma_{\ell}} \frac{1}{k^{1+\delta}}\left[\sum_{i=\tau_{1}}^{\sigma_{1}-1}\left\|u_{i}^{0}\right\|^{2}+\sum_{i=\sigma_{1}}^{\tau_{2}}\left\|u_{i}^{0}\right\|^{2}+\cdots\right. \\
& \left.+\sum_{i=\sigma_{\ell-1}}^{\tau_{\ell}}\left\|u_{i}^{0}\right\|^{2}+\sum_{i=\tau_{\ell}+1}^{k}\left\|u_{i}^{0}\right\|^{2}\right] \\
& \leq \sup _{\tau_{\ell} \leq k<\sigma_{\ell}} \frac{1}{k^{1+\delta}}\left[2 \sum_{i=1}^{\ell-1} \frac{\tau_{i+1}^{1+\delta}}{2^{i}}+\sum_{i=\tau_{\ell}+1}^{k}\left\|u^{0}\right\|^{2}\right] \\
& \leq 2+\sup _{\tau_{\ell} \leq k<\sigma_{\ell}} \frac{1}{k^{1+\delta}} \sum_{i=\tau_{\ell}}^{k}\left\|u_{i}^{0}\right\|^{2} \\
& \leq 2+\sup _{\tau_{\ell} \leq k<\sigma_{\ell}} \frac{1}{k^{1+\delta}}\left(k^{1+\delta}+\left\|u_{\tau_{\ell}}^{0}\right\|^{2}\right) \\
& \leq 3+\frac{1}{u_{\tau_{\ell}}^{0} \|^{2}} \leq 3+\frac{1}{\tau_{\ell}^{\ell+\delta}}<4 .
\end{aligned}
$$


Therefore, $\sum_{i=1}^{k}\left\|u_{i}^{s}\right\|^{2}=O\left(k^{1+\delta}\right)$ and theorem 2 again leads to the strong consistency of $B_{k}$.

By theorem 3.4 and remark 3.3 of Chen \& Guo (1991),

$$
S_{k} \rightarrow S, k \rightarrow \infty,
$$

from which it also follows that

$$
F_{k} \rightarrow F, \quad\left\|b_{k}-\hat{b}_{k}\right\| \rightarrow 0 \quad \text { and }\left\|d_{k}-\hat{d}_{k}\right\| \rightarrow 0, \quad k \rightarrow \infty .
$$

Since $F$ is a stable matrix and $F_{k} \rightarrow F$, there exists a $\rho \epsilon(0,1)$ and a $C>0$ such that

$$
\left\|F_{k} F_{k-1} \cdots F_{1}\right\| \leq C \rho^{k} \quad \forall k .
$$

We now show that there exists an $\ell_{0}$ such that

$$
\tau_{\ell_{0}}<\infty \quad \text { and } \sigma_{\ell_{0}}=\infty \text {. }
$$

If $\sigma_{\ell}<\infty$ and $\tau_{\ell+1}=\infty$ for some $\ell$, then $u_{i}^{*}=u_{i}^{d} \forall i \geq \sigma_{\ell}$ and from (49) it follows that $\left\{\hat{x}_{k}\right\}$ is bounded. Hence $\left\{u_{k}^{0}\right\}$ is bounded and by definition (56) $\tau_{\ell+1}$ must be finite. This means that $\sigma_{\ell}<\infty$ and $\tau_{\ell+1}=\infty$ cannot happen for any $\ell$.

If $\sigma_{\ell}<\infty$ and $\tau_{\ell}<\infty$ for all $\ell$, then for $i \epsilon\left[\tau_{\ell}, \sigma_{\ell-1}\right]$,

$$
\begin{aligned}
\hat{x}_{i+1} & =A \hat{x}_{i}+B_{i}\left(L_{i} \hat{x}_{i}+\hat{d}_{i}+u_{i}^{d}\right) \\
& =F_{i} \hat{x}_{i}+B_{i}\left(\hat{d}_{i}+u_{i}^{d}\right),
\end{aligned}
$$

so that

$$
\begin{aligned}
\hat{x}_{i+1}= & F_{i} F_{i-1} \cdots F_{\tau_{\ell}} \hat{x}_{\tau_{\ell}}+\sum_{j=1}^{i-\tau_{\ell}} F_{i} F_{i-1} \cdots F_{i-j+1} B_{i-j}\left(\hat{d}_{i-j}+u_{i-j}^{d}\right) \\
& +B_{i}\left(\hat{d}_{i}+u_{i}^{d}\right) .
\end{aligned}
$$

Using this and (61) we have

$$
\begin{aligned}
\left\|\hat{x}_{i+1}\right\| & \leq C \rho^{i-\tau_{\ell}+1}\left\|\hat{x}_{\tau_{\ell}}\right\|+C \sum_{1} \sum_{j=0}^{i-\tau_{\ell}} \rho^{j} \\
& =C \rho^{i-\tau_{\ell}+1}\left\|\hat{x}_{\tau_{\ell}}\right\|+C_{2},
\end{aligned}
$$

so that

$$
\left\|\hat{x}_{i+1}\right\|^{2} \leq 2 C \rho^{2\left(i-\tau_{\ell}+1\right)}\left\|\hat{x}_{\tau_{\ell}}\right\|^{2}+2 C_{2}^{2} .
$$

Therefore,

$$
\sum_{i=\tau_{\ell}-1}^{\sigma_{\ell}-1}\left\|\hat{x}_{i+1}\right\|^{2} \leq C_{3}\left\|\hat{x}_{\tau_{\ell}}\right\|^{2}+C_{4} \sigma_{\ell},
$$

or

$$
\sum_{i=\tau_{\ell}}^{\sigma_{\ell}}\left\|\hat{x}_{i}\right\|^{2} \leq C_{3}\left\|\hat{x}_{\tau_{\ell}}\right\|^{2}+C_{4} \sigma_{\ell}=o\left(\sigma_{\ell}^{1+\delta}\right) \text { as } \ell \rightarrow \infty .
$$


This result, along with (48), gives us

$$
\sum_{i=\tau_{\ell}}^{\sigma_{\ell}}\left\|u_{i}^{o}\right\|^{2}=o\left(\sigma_{\ell}^{1+\delta}\right) \quad \text { as } \ell \rightarrow \infty
$$

This means that for sufficiently large $\ell$.

$$
\sum_{i=\tau_{\ell}}^{\sigma_{i}}\left\|u_{i}^{0}\right\|^{2}<\sigma_{\ell}^{1+\delta}
$$

which contradicts the definition of $\sigma_{\ell}$, since by (55)

$$
\sum_{i=\tau_{\ell}}^{\sigma_{\ell}}\left\|u_{i}^{0}\right\|^{2}>\sigma_{k}^{1+\delta}+\left\|u_{\tau_{\ell}}^{0}\right\|^{2}
$$

Thus we cannot have $\tau_{\ell}<\infty$ and $\sigma_{\ell}<\infty$ for all $\ell$.

Consequently (62) holds and

$$
u_{k}^{*}=u_{k}^{0}+u_{k}^{d}
$$

and $\hat{x}_{k+1}=F_{k} \hat{x}_{k}+B_{k} \hat{d}_{k}, \quad \forall k \geq \tau_{\ell_{0}}$.

By (61) and the boundedness of $B_{k} \hat{d}_{k}$ it is clear that $\left\{\hat{x}_{k}\right\}$ is also bounded and so is $\left\{u_{k}^{0}\right\}$, so that

$$
\sum_{i=1}^{k}\left\|u_{i}^{s}\right\|^{2}=O(k)
$$

This and theorem 2 imply (60).

To prove optimality, notice that

$$
x_{k+1}-\hat{x}_{k+1}=A\left(x_{k}-\hat{x}_{k}\right)+\left(B-B_{k}\right) u_{k}^{*} .
$$

By the boundedness of $\left\{u_{k}^{*}\right\}$, the stability of $A$ and the fact that $B-B_{k} \rightarrow 0$ we find that

$$
x_{k}-\hat{x}_{k} \longrightarrow 0, k \rightarrow \infty \text {. }
$$

This, along with the boundedness of $\left\{\hat{x}_{k}\right\}$, implies that $\left\{x_{k}\right\}$ is also bounded. Therefore, $\left\{u_{k}^{*}\right\} \in \mathcal{U}$. Using (63) and the facts that $L_{k}-L \rightarrow 0$, and $\left\|d_{k}-\hat{d}_{k}\right\| \rightarrow 0$, we conclude that

$$
\frac{1}{N} \sum_{k=0}^{N-1}\left(u_{k}^{*}-L x_{k}-d_{k}\right)^{\prime}\left(Q_{2}+B^{\prime} S B\right)\left(u_{k}^{*}-L x_{k}-d_{k}\right) \longrightarrow 0, \quad k \rightarrow \infty .
$$

Combining this with (14) establishes (59).

\section{Conclusion}

We solved a class of stochastic adaptive control problems in the state space form which arise in controlling aircraft flying in gusty conditions. The important, although difficult, extensions which should be further looked into involve the state noise case and/or when the parameters $A$ and $C$ also contain unknown elements. The approach presented in this paper does not directly go over to this most general situation, but may possibly be used there in combination with some other techniques. 


\section{References}

Åström K J, Wittenmark B 1973 On self-tuning regulators. Automatica 9: 185-199

Bagchi A 1993 Optimal control of stochastic systems (London: Prentice Hall International)

Balakrishnan A V 1987 Kalman filtering theory (New York: Optimization Software)

Caines P, Lafortune S 1984 Adaptive control with recursive identification for stochastic linear systems. IEEE Trans. Autom. Control AC-29: 312-321

Chen H F 1984 Recursive system identification and adaptive control by use of the modified least squares algorithm. SIAM J. Control Optimization 22: 758-776

Chen H F, Guo L 1991 Identification and stochastic adaptive control (Boston: Birkhäuser)

Illiff K W 1973 Identification and stochastic control with application to flight control in turbulence. $\mathrm{Ph} \mathrm{D}$ thesis, University of California, Los Angeles

Kumar P R 1983 Optimal adaptive control of linear quadratic Gaussian systems. SIAM J. Control Optimization 21: 163-178

Kumar P R 1985 A survey of some results in stochastic control. SIAM J. Control Optimization 23: $329-380$

Polderman J W 1989 Adaptive control and identification: Conflict or conflux? CWI Tract 67, (Amsterdam: Centre for Mathematics \& Computer Science)

Wang G H 1993 A stochastic adaptive control application to flight systems. Ph D thesis, University of California, Los Angeles 